\title{
Nouveau contexte environnemental et réglementaire : quel impact pour la culture du tournesol ?
}

\author{
Étienne PILORGÉ \\ CETIOM, \\ Direction scientifique, \\ Campus de Grignon, \\ Avenue Lucien-Brétignières, \\ F-78850 Thiverval-Grignon, \\ France \\ <pilorge@cetiom.fr>
}

\begin{abstract}
This paper tries to analyze the influences of changes in the environmental and regulatory context on the perspectives of development of sunflower in France and the European Union. From the perspective of the European Directive 2009/28/EC on renewable energy, results of sunflower in French conditions, for energy balance and greenhouse gas emission, appear quite satisfying. In a context of reduced uses of plant protection products, sunflower crop presents some advantages because of the strong investment in the past with respect to tolerance to diseases and pests. Where scenarios of limitation of summer irrigation would repeat, the sunflower could be promoted. Sunflower has the characteristic of being few demanding in mineral fertilizer. All these points show than sunflower presents many advantages with respect to recent developments in context, which are making more necessary than ever research efforts for improvement of yields.
\end{abstract}

Key words: sunflower context, SWOT, biofuels, nitrate directive, water use, pesticides regulation
Avec environ 710000 ha en 2010, les surfaces de tournesol françaises retrouvent un niveau oublié depuis le début des années 2000, en nette progression par rapport aux 515000 ha de 2007. Au niveau européen, le tournesol est également une culture bien représentée avec près de 3,75 millions d'hectares, principalement en Roumanie, en Bulgarie, en Espagne, en France, et en Hongrie. Avec des rendements relativement satisfaisants, la France était le deuxième producteur derrière la Hongrie en 2008.

Au niveau des marchés, l'ensemble des cultures oléagineuses jouit aujourd'hui d'une bonne situation relative dans un contexte agricole globalement déprimé après le violent repli à la suite des pics de 2008, avec des rapports de prix de l'ordre de 2,5 par rapport au blé (mais seulement 1,7 à 1,8 par rapport au maïs), à même de leur assurer une certaine compétitivité dans les systèmes de culture français. Les cours des oléagineux ont relativement résisté, malgré des baisses de 35 à $45 \%$ par rapport aux sommets spéculatifs de 2008. Une demande alimentaire soutenue et en croissance mais également le développement des biocarburants à base d'huiles végétales expliquent cette situation. Quelles influences peuvent avoir les évolutions du contexte environnemental et réglementaire sur la relative bonne santé de cet oléagineux du Sud?

\section{En matière de biocarburants, le tournesol présente un bon niveau de durabilité}

L'EEB (European Biodiesel Board) estimait la production européenne de biodiesel à 7,7 millions de tonnes (MT) en 2008, soit $65 \%$ du biodiesel mondial, qui représenterait de l'ordre de $12 \mathrm{MT}$ en 2008. Au regard de la production mondiale d'huile, ces quantités sont loin d'être négligeables : I'utilisation de biodiesel de I'UE représente de l'ordre du tiers de sa consommation $\mathrm{d}^{\prime}$ huile, et le biodiesel mondial près de $10 \%$ de la production mondiale d'huiles végétales, mais de l'ordre de $19 \%$ des échanges internationaux (environ 61 MT en 2009-2010 selon Oil World). Le biodiesel occupe désormais une place non négligeable à même d'influer le marché des huiles. Toutefois, les prévisionnistes ne tablent pas sur une expansion effrénée du biodiesel, d'une part du fait des résistances des pétroliers et de l'industrie automobile (normes sur les carburants), d'autre part du fait des réglementations européennes sur la durabilité des biocarburants.

Les évolutions de ces réglementations concernant les biocarburants seront déterminantes pour le développement de ces marchés et des filières de production. Si la directive européenne sur les énergies renouvelables 2009/28/EC demande à ce qu'au moins $10 \%$ de la consommation énergétique du secteur des transports des pays de I'UE proviennent d'énergies renouvelables - dont les biocarburants font partie - en 2020, elle impose également que leur production s'inscrive dans un cadre durable et réponde à une série de critères précis, dont ceux, majeurs, portant sur leur bilan énergétique et leur bilan d'émissions de gaz à effet de serre (GES), qui sont au fondement de leur légitimité. Les filières de production devront prouver que le biocarburant permet une réduction de $35 \%$ des émissions de GES par rapport à son équivalent fossile, ce ratio devant être au minimum $50 \%$ à partir de 2017. La problématique de l'évaluation des filières de production à l'aide d'analyses de cycle de vie devenant cruciale, I'ADEME (Agence de l'environnement et de la maîtrise de l'énergie) s'est attachée à fournir « un référentiel méthodologique $d^{\prime}$ analyse des impacts des filières biocarburants sur les GES et les consommations d'énergie liés à ces filières au niveau français » élaboré de façon consensuelle, et a commandité une étude publiée en février 2010.

Les résultats concernant la filière tournesol dans les conditions françaises apparaissent tout à fait honorables dans cette étude : la comparaison des impacts environnementaux du biodiesel de tournesol et du diesel, sur la base de leurs valeurs énergétiques en mégajoule, fait apparaître une réduction de $68 \%$ de la consommation d'énergie primaire non renouvelable et de $73 \%$ des émissions de GES (les équivalents pour la filière biodiesel colza étant de 65 et $59 \%$ respectivement, de 69 et $77 \%$ pour la filière biodiesel soja, de 78 et $76 \%$ pour la filière biodiesel palme). La filière agricole de référence de l'éthanol de canne à sucre offre des réductions de 85 et $72 \%$, respectivement. La filière biodiesel tournesol offre donc sur ces critères des résultats parmi les meilleurs des filières de biocarburants européens et répond aux critères de la directive de l'UE sur les GES. Elle présente donc des atouts de durabilité et des bilans très satisfaisants, malgré des performances de productivité au champ qui restent 
limitées et fort en deçà du potentiel de cette culture. Moyennant une intensification raisonnée de la culture, notamment des apports d'eau limités dans les situations qui le méritent, ces bilans encourageants pourraient encore être améliorés.

Enfin, il faut signaler que le progrès génétique et la demande aidant, près de $50 \%$ des surfaces sont aujourd'hui cultivées en tournesol oléique, préféré pour les usages en biocarburants et en oléochimie.

\section{Quatrième programme d'action de la directive nitrates}

La déclinaison française du quatrième programme d'action de la directive nitrates indique deux objectifs principaux en zones vulnérables, I'un généralisant les couverts enherbés ou pérennes le long des cours d'eau, l'autre visant une couverture du sol en période de risque de lessivage de $100 \%$ des surfaces cultivées au plus tard à l'échéance de 2012. Ce second point est particulièrement important pour les cultures de printemps en général, et le tournesol en particulier. En effet, l'implantation d'un couvert sur la période hivernale pose de nombreuses questions techniques, $d^{\prime}$ une part quant à sa destruction pour permettre la préparation du sol et l'implantation du tournesol dans de bonnes conditions, d'autre part quant au choix de l'espèce utilisée en couverture, de manière à produire un état sanitaire satisfaisant en termes de maladies et de ravageurs et à assurer sinon un enrichissement du sol en azote, du moins une dynamique de l'azote satisfaisante pour la culture de printemps. Les modalités de réalisation des couverts (espèces possibles, dates de destruction) sont encadrées par la réglementation et ses déclinaisons régionales, et la souplesse parfois apportée par des régimes dérogatoires devrait disparaître d'ici 2012. De fait, la gestion des couverts et leur destruction constituent un défi technique important pour les praticiens et concernent l'ensemble des cultures de printemps. Les déclinaisons locales de ces réglementations et la qualité des solutions techniques qui seront mises au point sont susceptibles $d$ 'influer sur la performance future des cultures de tournesol.

\section{Grenelle de l'environnement et ECOPHYTO 2018}

La loi Grenelle 1 du 5 août 2009 fixe l'objectif de « réduire de moitié les usages des produits phytopharmaceutiques et des biocides en dix ans ». Si ses modalités d'application font encore l'objet de débats, le cap est bel et bien donné et la mise en place du schéma de gouvernance et de mise en œuvre du plan ECOPHYTO 2018 est en cours aujourd'hui.

Dans un contexte de rareté - réglementaire ou liée à l'innovation - de l'intrant phytopharmaceutique, le tournesol est une culture plutôt avantagée du fait du fort investissement passé en matière de tolérance aux maladies et d'une faune de ravageurs limitée en dehors des oiseaux, des gibiers et des limaces.

L'étude ECOPHYTO R\&D menée par I'INRA a calculé, sur la base des enquêtes CETIOM, un indice de fréquence de traitement (IFT) moyen de 2,1 pour la culture du tournesol, dont 1,6 pour la fraction herbicide, ce qui la classe avec le maïs parmi les grandes cultures les moins utilisatrices de produits phytopharmaceutiques au vu des pratiques actuelles, mais également, selon les " dires d'experts ", les mieux à même de s'adapter à une diminution d'utilisation par rapport à des pratiques qualifiées $d^{\prime}$ 'intensives sans forte baisse de rendement.

Les enquêtes CETIOM 2009 montrent que le principal poste de protection phytosanitaire est le désherbage : $36 \%$ des surfaces reçoivent un produit unique, $56 \%$ reçoivent deux produits différents. Par ailleurs, $28 \%$ des surfaces reçoivent un herbicide total pendant l'interculture.

Quatre-vingt-dix-huit pour cent des parcelles françaises sont désherbées en plein. Toutefois, en région Bourgogne, où un fort effort de développement des techniques de désherbage « mixtes » associant chimique et mécanique a été réalisé, $7 \%$ des parcelles sont désherbées avec des applications localisées dans le rang uniquement, l'interrang étant en général biné mécaniquement. Cette technique permet de diviser la quantité d'herbicide utilisée par un facteur de 2 à 3 . En Champagne (où la méthode est plus anciennement connue sur betteraves), le taux est de 4,5\%. Au niveau national, le binage est pratiqué sur $40 \%$ des surfaces, le plus souvent en complément d'herbicides appliqués en plein. Ces chiffres montrent, à la fois, que cette technique est faisable au moins dans certaines conditions de systèmes d'exploitation (main-d'œuvre et matériel doivent être disponibles au moment opportun) et maîtrisée par une partie des agriculteurs, et que le tournesol conserve une marge de progrès quant à I'utilisation des herbicides.

La même enquête CETIOM 2009 montre que seules $10 \%$ des surfaces nationales ont reçu un traitement fongicide en végétation en 2009 , et $11 \%$ un insecticide (après $40 \%$ en 2006 et $60 \%$ en 2004). Soixante-huit pour cent des parcelles ont été traitées contre les limaces au moins une fois, avec des fréquences de traitement relativement stables depuis 2002, contrairement à ce qui est observé pour les insecticides.
Dans un contexte de réduction de l'utilisation des produits phytosanitaires, la tentation pourrait être, pour faire baisser I'IFT moyen des exploitations, d'augmenter la sole des cultures peu consommatrices, ce qui avantagerait le tournesol. Cette stratégie présente toutefois au moins deux limites : d'une part, la saturation des assolements et des rotations avec une culture s'accompagne généralement de pressions phytosanitaires accrues, qui pourraient s'avérer dangereuses à terme, d'autre part l'agriculture produit pour un marché sur lequel les matières premières ne sont pas complètement substituables.

Dans un contexte de diminution de l'usage à la culture, l'effort en tournesol devra porter prioritairement sur les herbicides. L'utilisation du désherbage mécanique seul sera difficilement généralisable dans des contextes de surfaces importantes par exploitation (grandes structures) du fait des temps de travaux et des risques de mauvaise maîtrise de la flore. Progresser sur ce point demandera donc d'innover. Deux voies semblent d'ores et déjà ouvertes. La voie technologique utilisant des outils de binage guidés de façon automatique (par GPS ou capteurs embarqués) et permettant de gros débits de chantiers, voie valorisable tant en systèmes classiques qu'en systèmes biologiques. La voie du désherbage mixte offre une marge de progrès importante mais se trouve fortement limitée par l'absence de produits herbicides de postlevée. Ce frein sera levé par l'arrivée sur le marché de variétés de tournesols tolérantes à des matières actives de postlevée (l'imazamox ou le tribénuron-méthyl), variétés non OGM sélectionnées par la technique éprouvée de la mutagenèse. Cette possibilité de contrôler la flore en postlevée supprime en grande partie le risque de ne pas pouvoir contrôler la flore adventice à la suite d'une impasse en présemis ou en prélevée - impasse totale ou partielle dans le cas du désherbage mixte - et de la conjonction de circonstances climatiques défavorables au binage. À l'extrême, si aucune infestation significative n'est observée, il devient possible de ne plus traiter du tout sans prise de risque conséquente. Dès lors que les variétés portant cette innovation présenteront un bon niveau sur l'ensemble des critères (rendement, tolérance aux maladies, profils $d^{\prime}$ acides gras, teneurs en huiles, etc.), cette innovation paraît susceptible de contribuer au développement de techniques alternatives ou mixtes en systèmes de production traditionnels en offrant une sécurité jusqu'alors inexistante.

\section{Contraintes sur l'eau}

Ces dernières années, marquées par les sécheresses estivales, ont vu dans certaines régions une multiplication des arrêtés administratifs 
limitant ou interdisant l'irrigation en cours d'été. Dans ce contexte d'incertitude quant à la possibilité d'irriguer, liée à la météorologie de l'année, le tournesol présente le double avantage d'un comportement satisfaisant en conditions hydriques limitantes et d'une bonne réponse à des quantités d'eau limitées. Le plus souvent cultivé en sec, il trouve également sa place sur les exploitations disposant d'irrigation: en effet, sa présence permet d'équilibrer l'assolement avec une culture peu gourmande en eau, et donc de réduire la quantité d'irrigation globale, mais également de bien valoriser des quantités d'eau limitées : les références CETIOM montrent qu'en conditions sèches en terres superficielles, $80 \mathrm{~mm}$ d'eau en deux apports à partir de la floraison permettent de gagner de l'ordre de $8 \mathrm{q} /$ ha et $2 \%$ d'huile. Dans le cas où les scénarios de limitation de l'irrigation estivale se répéteraient, le tournesol pourrait se trouver favorisé.

\section{Tournesol et agriculture biologique}

La loi Grenelle 1 d'août 2009 fixe l'objectif de $6 \%$ de la SAU française en agriculture biologique en 2012 et de $20 \%$ en 2020. Par ailleurs, le marché de l'alimentation bio croît de l'ordre de $10 \%$ par an quand le marché global de l'alimentation croît de 3,6 \% par an, et le manque de matière première est estimé à $20 \%$ sur une base tournesol, colza et soja: une marge de croissance importante, bien que modeste en valeur absolue, existe. Le tournesol bio ou en conversion vers le bio représentait 10055 ha (source Agence Bio) en 2008, soit 1,6\% de la sole oléagineuse nationale (le bio représente moins de 0,1\% pour le colza, et 0,9\% pour les céréales). Ce résultat, très honorable à l'aune des grandes cultures, s'explique par ses atouts en système de production biologique, notamment une gamme variétale offrant de bons niveaux de tolérance aux maladies, bénéfice de près de 40 ans d'investissement des acteurs de la filière et de la recherche publique, et une structure de peuplement parfaitement adaptée au contrôle des adventices par les méthodes mécaniques. Les ravageurs sont quasi absents en végétation, mais la phase d'implantation est sensible aux dégâts de limaces, d'insectes du sol et d'oiseaux contre lesquels il n'existe pas de méthodes de lutte efficaces. En matière de fertilisation azotée, facteur globalement limitant en agriculture biologique, le tournesol présente la caractéristique d'être peu gourmand en fertilisation minérale du fait d'un système racinaire très efficace. Le revers en est sa réputation d'épuiser le profil... Sa place dans la rotation doit donc être soigneusement choisie, sans sous-estimer le fait que malgré la rusticité de cette culture, l'azote peut être limitant aussi pour le tournesol. D'autres travaux de R\&D permettraient sans doute d'améliorer ses performances dans ce contexte : choix variétal, place dans la rotation, stratégies de lutte contre les adventices, par exemple. Reste que le tournesol, avec le soja, est certainement l'oléagineux annuel le mieux adapté à l'agriculture biologique.

Nous constatons donc que le tournesol présente de nombreux atouts vis-à-vis des évolutions récentes du contexte et, qu'à un certain degré, ce contexte lui devient plus favorable. Sa principale limite actuellement réside dans la stagnation des rendements au champ, en moyenne stationnaires depuis plusieurs années. Les origines de cette situation semblent résider à plusieurs niveaux: le positionnement de cette culture à image « rustique » dans des conditions plutôt difficiles et des terres de moindre potentiel, les effets plus marqués des sécheresses estivales, et l'impact de certaines maladies, le phoma notamment. Les efforts de recherche actuels, qui font l'objet d'une collaboration fortement structurée autour des programmes de l'UMT tournesol de Toulouse, et également des efforts des sélectionneurs, visent à relever ce défi de la stagnation et de l'irrégularité des rendements. Dans le contexte actuel, des succès de recherche ont toutes les chances de se traduire aux champs.

\section{RÉFÉRENCES}

http://www.biofuelstp.eu/.

Analyses de cycle de vie appliquées aux biocarburants de première génération consommés en France. Rapport final de l'étude ADEME, février 2010.

Oil World annual 2009 vol 2.

L'agriculture biologique. Chiffres clés. Agence Bio. Édition, 2009.

Loi n ${ }^{\circ} 2009-967$ du 3 août 2009 de programmation relative à la mise en œuvre du Grenelle de l'environnement (1). Journal officiel de la République française du 5 août 2009.

Synthèse du rapport de l'étude ECOPHYTO R\&D, INRA janvier 2010.

Merrien A. Tournesol, une réelle opportunité pour vos assolements irrigués. Perpectives agricoles $n^{\circ} 367$, mai 2010 : 73-4. 